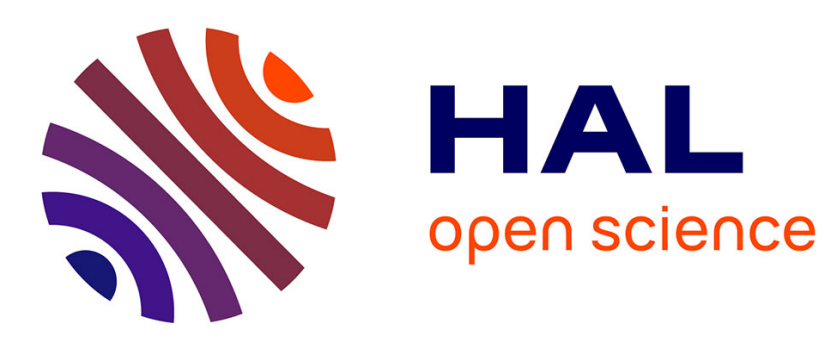

\title{
Probabilistic impedance of foundation: Impact of the seismic design on uncertain soils
}

\author{
R. Cottereau, Didier Clouteau, Christian Soize
}

\section{To cite this version:}

R. Cottereau, Didier Clouteau, Christian Soize. Probabilistic impedance of foundation: Impact of the seismic design on uncertain soils. Earthquake Engineering and Structural Dynamics, 2008, 37 (6), pp.899-918. 10.1002/eqe.794 . hal-00685116

\section{HAL Id: hal-00685116 https://hal.science/hal-00685116}

Submitted on 4 Apr 2012

HAL is a multi-disciplinary open access archive for the deposit and dissemination of scientific research documents, whether they are published or not. The documents may come from teaching and research institutions in France or abroad, or from public or private research centers.
L'archive ouverte pluridisciplinaire HAL, est destinée au dépôt et à la diffusion de documents scientifiques de niveau recherche, publiés ou non, émanant des établissements d'enseignement et de recherche français ou étrangers, des laboratoires publics ou privés. 


\title{
Probabilistic impedance of foundation: impact on the seismic design on uncertain soils
}

\author{
Régis Cottereau $^{1, *}$, Didier Clouteau ${ }^{1}$, Christian Soize ${ }^{2}$ \\ 1 École Centrale Paris, Laboratoire MSSMat, Grande Voie des Vignes, 92295 Châtenay-Malabry, France \\ 2 Université Paris-Est, Laboratoire de Mécanique, 5 bd Descartes, 77454 Marne-la-Vallée, France
}

\begin{abstract}
SUMMARY
In the linear seismic design of buildings, the (deterministic) substructure method is a customary and efficient approach. However, the existence of spatial variability in the parameters of the mechanical model of the soil, as well as parametric errors, call for the use of probabilistic approaches in order to provide a reliable design of the structure. The construction of probabilistic models of the soil impedance matrix provides a natural path to such approaches within the context of the substructure method. Two main techniques are described in this paper: a parametric one, typically using the Stochastic Finite Element method, and a nonparametric one, that was introduced more recently. The latter is explored more specifically, and the possibilities it offers in terms of seismic design are presented. In particular, it is shown that it allows for the estimation of quantiles of the quantities of interest, rather than confidence intervals, which lead to highly conservative design. Copyright (c) 2007 John Wiley \& Sons, Ltd.
\end{abstract}

KEY WORDS: Soil impedance matrix; Probabilistic design; Lumped-parameter model; Substructure method; Stochastic Finite Element Method; Nonparametric probabilistic method.

\section{INTRODUCTION}

In the linear seismic design of buildings, the substructure method is a customary and efficient approach [1, 2]. It consists in considering the problem in the frequency domain, and to take into account the dynamical influence of the soil through its impedance matrix. Because of the numerical complexity, and of a general lack of data, this soil impedance matrix is often computed with a simplifying hypothesis of homogeneous horizontal layers. However, the spatial variability of its parameters within each layer may induce important local effects, like differential displacements under the foundation, and should be quantified. Beside this model simplification, large errors might also appear during the identification of the parameters of the soil model, and these parametric errors may in turn lead to large drifts in the overall design.

Stochastic approaches $[3,4,5]$ provide a mean to take into account these model and parametric errors in the design of the building. Within the context of the substructure method,

\footnotetext{
*Correspondence to: regis.cottereau@ecp.fr
} 


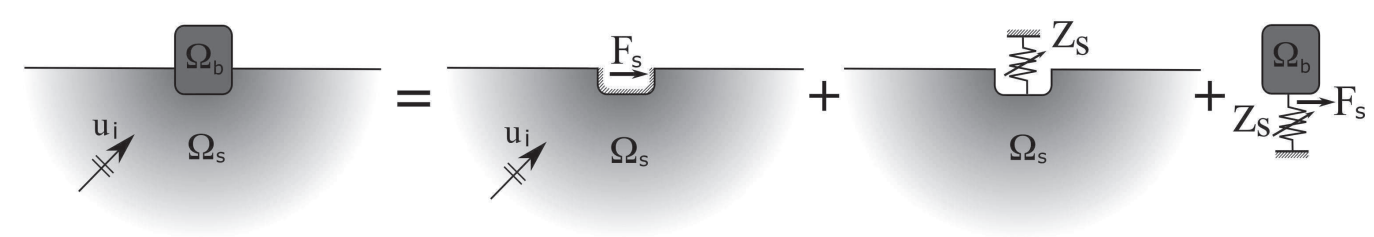

Figure 1. The substructure method: the global problem (on the left) decomposed in a local problem on the soil $\Omega_{s}$ to define the equivalent seismic loading vector (second picture) and the soil impedance matrix (third picture), and a global problem on the building $\Omega_{b}$ and the interface $\Gamma$.

this means constructing a probabilistic model of the building, of the seismic loading, and of the soil impedance matrix. We will concentrate in this paper on the latter, which has been less treated in the literature. The main objective, here, is to investigate the influence of the soil uncertainty on the overall design of the building, in particular with respect to high quantiles of the quantities of interest, rather than the often-encountered confidence intervals. Classical parametric approaches do not seem appropriate in that matter, as they are usually numerically very expensive. Besides, they require the definition of random models for the soil parameters, while very little data is usually available and cannot take into account model errors that are typically very important in geomechanics. However, a nonparametric approach was recently introduced, and will be shown in this paper to be particularly appropriate for seismic design on uncertain soils. It should noted that the relative influences of the uncertainty on the soil, the structure, and the seismic load, will not be discussed in this paper. The structure and the load will be modeled throughout as deterministic.

In the next section, the classical deterministic substructure method is recalled, with the possible contributions from probabilistic approaches, and the corresponding quantities of interest for the seismic design of a structure. In particular, the quantiles and confidence intervals for these quantities will be defined and compared. The following two sections concentrate, respectively, on the description of the deterministic and probabilistic models of the soil impedance matrix. For the latter, the nonparametric approach is presented along with an extensive review of the parametric approaches found in the literature. Finally, in section 5, the deterministic model of a building and of a seismic signal are presented and some aspects of the seismic design of the building are discussed, highlighting the influence of the uncertainty in the soil.

\section{THE SOIL-STRUCTURE INTERACTION PROBLEM}

\subsection{The (deterministic) substructure method}

We consider here a classical soil-structure interaction (SSI) problem, where a building, denoted by "b", resting on a soil, denoted by "s", is loaded by an incoming seismic wave. The interface between the soil and the building, denoted by " $\Gamma$ ", may be flexible or rigid, and the building may be embedded in the soil or not. The classical substructure method $[1,2]$ consists in splitting the entire soil-structure domain $\Omega$ into two subdomains $\Omega_{s}$ and $\Omega_{b}$ connected through their common boundary $\Gamma$, and to replace the global problem by a local problem in the soil $\Omega_{s}$, where the soil impedance matrix $\left[Z_{s}(\omega)\right]$ and the equivalent seismic load $\left[F_{s}(\omega)\right]$ are computed, and 
one global problem, where $\left[Z_{s}(\omega)\right]$ and $\left[F_{s}(\omega)\right]$ are used as boundary conditions on $\Gamma$ (figure 1 ). In the following, we introduce a set of displacement fields $\left\{\boldsymbol{\Psi}_{\ell}\right\}_{1<\ell<n_{\Gamma}}$, defined on the soilstructure interface, with $n_{\Gamma}$ the number of Degrees-of-Freedom (DOFs) on the interface. In the case of a rigid foundation, $n_{\Gamma}=6$, and these fields correspond to the 6 rigid-body displacement fields of the interface.

In the soil subdomain $\Omega_{s}$, the elements of the soil impedance matrix $\left[Z_{s}(\omega)\right]$ and of the equivalent seismic loading vector $\left[F_{s}(\omega)\right]$ are defined, at each frequency $\omega$ in $\mathbb{R}$, and for $1 \leq k, \ell \leq n_{\Gamma}$, by

$$
\begin{gathered}
{\left[Z_{s}(\omega)\right]_{k \ell}=\int_{\Gamma} \mathbf{t}_{s}\left(\mathbf{v}_{\ell}(\omega)\right) \cdot \mathbf{\Psi}_{k} d S} \\
{\left[F_{s}(\omega)\right]_{k}=-\int_{\Gamma} \mathbf{t}_{s}\left(\mathbf{u}_{\mathrm{inc}}(\omega)+\mathbf{u}_{\mathrm{d}}(\omega)\right) \cdot \mathbf{\Psi}_{k} d S,}
\end{gathered}
$$

where the dot symbol $(\cdot)$ represents the hermitian scalar product on $\mathbb{C}^{3}, \mathbf{v}_{\ell}(\omega)$ is the radiated field in the soil corresponding to an imposed displacement $\Psi_{\ell}$ on $\Gamma, \mathbf{t}_{s}\left(\mathbf{v}_{\ell}(\omega)\right)$ is the traction vector field on $\Gamma$ corresponding to the radiated field $\mathbf{v}_{\ell}(\omega)$ in the soil, $\mathbf{u}_{\text {inc }}(\omega)$ is the incident displacement field in the soil, modeling the incoming seismic wave, and $\mathbf{u}_{\mathrm{d}}(\omega)$ is the scattered displacement field in the soil, corresponding to the scattering of the incoming wave on the interface, which is then supposed fixed.

In the building, a set of $n \geq n_{\Gamma}$ displacements fields is defined on $\Omega_{b}$, compatible with $\left\{\boldsymbol{\Psi}_{\ell}\right\}_{1 \leq \ell \leq n_{\Gamma}}$ along the soil-structure interface $\Gamma$. Classically, the $n_{\Gamma}=6$ rigid-body fields of the building are considered, and they are completed with $n_{b}$ eigenmodes of the building on a clamped base. The classical reduced matrices of mass $\left[M_{b}\right]$, damping $\left[D_{b}\right]$ and stiffness $\left[K_{b}\right]$ of the building are then defined with respect to these $n$ displacement fields. The equation of motion in $\Omega_{b}$ then reads

$$
\left.\left(\left[\begin{array}{cc}
{\left[Z_{s}(\omega)\right]} & {\left[0_{\Gamma b}\right]} \\
{\left[0_{\Gamma b}\right]^{T}} & {\left[0_{b b}\right]}
\end{array}\right]-\omega^{2}\left[M_{b}\right]+i \omega\left[D_{b}\right]+\left[K_{b}\right]\right)\left[\begin{array}{c}
{\left[q_{\Gamma}(\omega)\right.} \\
{\left[q_{b}(\omega)\right.}
\end{array}\right]\right]=\left[\begin{array}{c}
{\left[F_{s}(\omega)\right]} \\
{\left[0_{b 1}\right]}
\end{array}\right]
$$

where $\left[q_{\Gamma}(\omega)\right]$ are the coefficients of the displacement field in the building corresponding to the $n_{\Gamma}$ DOFs inducing a displacement of the foundation, $\left[q_{b}(\omega)\right]$ are those corresponding to the $n_{b}$ DOFs on fixed base, and $\left[0_{\Gamma b}\right],\left[0_{b b}\right]$ and $\left[0_{b 1}\right]$ are matrices with zero-valued entries, respectively of size $n_{\Gamma} \times n_{b}, n_{b} \times n_{b}$, and $n_{b} \times 1$.

It is customary to introduce a kinematic interaction coefficient $\left[c_{o}(\omega)\right]$, defined by

$$
\left[F_{s}(\omega)\right]=\left[Z_{s}(\omega)\right]\left[c_{o}(\omega)\right]
$$

which represents the movement that the foundation would undergo, were it to be massless and rid of the building. When considering a vertically-propagating plane wave, a horizontallylayered soil, and a rigid surface foundation, there is no kinematic interaction because the movement induced by the wave is compatible with the geometry of the foundation. In that case, $\left[c_{o}(\omega)\right]$ describes directly the incident wave field and the equation of motion (3) can be written in terms of DOFS relative to the foundation in a frame moving with $\left[c_{o}(\omega)\right]$ :

$$
\left(\left[\begin{array}{cc}
{\left[Z_{s}(\omega)\right]} & {\left[0_{\Gamma b}\right]} \\
{\left[0_{\Gamma b}\right]^{T}} & {\left[0_{b b}\right]}
\end{array}\right]+\left[S_{b}(\omega)\right]\right)\left[\begin{array}{c}
{[c(\omega)]} \\
{\left[q_{b}(\omega)\right]}
\end{array}\right]=-\left[S_{b}(\omega)\right]\left[\begin{array}{c}
{\left[c_{o}(\omega)\right]} \\
{\left[0_{b 1}\right]}
\end{array}\right],
$$

with $[c(\omega)]=\left[q_{\Gamma}(\omega)\right]-\left[c_{o}(\omega)\right]$, and $\left[S_{b}(\omega)\right]$ the dynamic stiffness of the building, defined by $\left[S_{b}(\omega)\right]=-\omega^{2}\left[M_{b}\right]+i \omega\left[D_{b}\right]+\left[K_{b}\right]$. 


\subsection{Probabilistic approaches}

In the modeling of this deterministic SSI problem, two types of controversial hypotheses are usually made:

1. Hypotheses on the model, with, in particular,

- The natural heterogeneity of the soil is modeled by a system of homogeneous layers [6];

- The seismic input is modeled by a vertically incident system of plane waves;

2. Hypotheses on the value of the parameters of the model, with, in particular,

- The mechanical and geometrical parameters of the soil layers are supposed to have been well identified;

- The amplitude of the seismic input is supposed to be perfectly known;

- The mechanical parameters of the building are supposed to have been well identified.

The assumed linearity of the behavior of the soil and of the soil-structure interface might also seem preposterous. However, in practice, this only limits the applicability of the substructure method and can be taken into account, to some extent, by using an hysteretic damping coefficient (see section 2.4).

With respect to the actual soil and the actual seismic wave, the first group of assumptions leads to model errors, while the other one corresponds to parameter uncertainties [7]. They induce an overall uncertainty on the evaluation of the response of the building, and hence an uncertainty on the design of the building. The goal of probabilistic approaches is to provide a mean to quantify this uncertainty, and hence to allow for a more rational design. The basic idea is to contemplate many "possible" situations, each relaxing the hypotheses seen above, and to assign weights for the outcome of each of these situations in the global design analysis. The differences between the available probabilistic methods lie in the choice of a) the set of the "possible" situations, and b) in the way the weights are assigned in the global design.

Of the hypotheses described above, those concerning the building $[8,9,3]$ and the seismic input $[4,10,11,12]$ have received earlier and wider attention. In particular, the spatial variability of $\left[F_{s}(\omega)\right]$, or equivalently $\left[c_{o}(\omega)\right]$, was extensively studied and led to the choice of space-time random fields models. We concentrate in this paper on the assumptions related to the soil model, that induce uncertainty on the soil impedance matrix $\left[Z_{s}(\omega)\right]$. Ultimately, the complete probabilistic treatment of seismic design problems would require the construction of probabilistic models for both $\left[F_{s}(\omega)\right]$ and $\left[Z_{s}(\omega)\right]$, addressing in particular the issue of their correlation.

It should be noted that probabilistic analyses are usually much more involved than their deterministic counterparts. Hence, in civil engineering, they are mainly restricted to the most sensitive projects, such as the construction of nuclear power plants [13, 14, 15]. However, for all structures on extended footings, as well as for multiply supported structures such as bridges, the consideration of the spatial variability of the soil properties (and of the seismic excitation) is essential for a rational design. 


\subsection{Quantities of interest}

In deterministic seismic design, the quantities of interest are the maximum strains and stresses in the structural elements, which are directly derived from the displacement and acceleration histories of the DOFs of the building, obtained by the resolution of equation (5). For the design of non-structural elements, pseudo-acceleration floor spectra are also used [17]. They give, at each frequency, an approximation of the maximum acceleration of a 1-DOF system submitted to a bottom acceleration imposed by the structure at the considered floor. Hence, they can be used to design those of the non-structural elements that can be modeled as 1DOF systems and do not interact with the structure, in the frequency range of interest in earthquake engineering. The use of floor spectra can also be extended for the design of nonstructural multi-DOFs systems when design rules such as the CQC modal sum [17] can be applied.

In probabilistic design, the quantities of interest are the statistical relatives of the previous ones. Indeed, as we consider a large number of "possible" structures, the design arising from the consideration of one particular realization of the random soil is meaningless. For a design random variable $X$, the mean $E\{X\}$ and standard deviation $\sigma_{X}=\sqrt{E\left\{(X-E\{X\})^{2}\right\}}$ are often considered in the literature. However, they only give a partial information on the behavior of this design quantity, respectively a trend, and a scattering of the results around that trend. With respect to the design of civil engineering structures, quantiles appear much more interesting. The $p$-quantile of a random variable $X$ is defined as the value $x_{p}$ such that the cumulative density function $F_{X}$ of $X$ verifies

$$
F_{X}\left(x_{p}\right)=P_{X}\left(X<x_{p}\right)=p .
$$

For the seismic design of strategic buildings, in particular, rather high quantiles, such as the 95\%-, or the 99\%-quantile, should be considered. The main difficulty with these quantiles is their estimation with the Monte Carlo method [18], because it requires the consideration of a (very) large number of trials. When computational costs are an issue, they are therefore often replaced by confidence intervals. For any (second-order [19]) random variable, an estimation of the $p$-confidence interval is available through Chebychev's inequality, stating that

$$
P_{X}\left(|X-E\{X\}| \leq \frac{\sigma_{X}}{\sqrt{1-p}}\right) \geq p .
$$

The confidence intervals estimated using equation (7) are rather loose, but their computation requires only the estimation of the mean and standard deviation of $X$. All the statistical quantities that were defined here for random variables can be extended for random processes depending on the frequency simply by considering the values at each frequency point independently.

It should be noted that the relation between the properties of the soil and the design variables is, in general, nonlinear. Therefore the mean value of a design variable is usually different from the value of the design variable corresponding to the mean values of the soil properties, sometimes quite significantly. For the same reason, the design method that consists in taking extreme values of the soil properties (usually $2 / 3$ and $3 / 2$ in earthquake engineering) and computing the corresponding values of the design variables as bounds of that design variable is inadequate. However, as it is often encountered in practice, we will show the results corresponding to these extreme values in our design case (section 5), and we will refer to this design method as the "extremal" design. 


\subsection{Normalization of the soil impedance matrix}

It is customary in SSI problems to normalize the soil impedance matrix as:

$$
\left[Z_{s}(\omega)\right]=\left[K_{0}\right]^{1 / 2}\left(\left[K_{s}(\omega)\right]+i\left[C_{s}(\omega)\right]\right)\left[K_{0}\right]^{T / 2},
$$

where $\left[K_{0}\right]$ is a $6 \times 6$ real diagonal matrix whose elements are approximate static values for the elements of the diagonal of the soil impedance matrix, and depend on the type of problem (e.g. embedded, surface, strip foundation) and the mechanical properties of the top layer of the soil. They are defined for example in $[20,21]$. $\left[K_{s}(\omega)\right]$ is the dynamic stiffness coefficient, $\left[C_{s}(\omega)\right]$ is the dynamic damping coefficient, both $6 \times 6$ real matrices, and such that $\left[C_{s}(\omega=0)\right]$ vanishes. This linear formulation is sometimes corrected by considering a diagonal $6 \times 6$ real hysteretic damping matrix $[B]$ such that, in equation $(8),\left[K_{0}\right]$ is replaced by

$$
\left[K_{0}^{*}\right]=\left[K_{0}\right]\left(\left[I_{6}\right]+2 i[B]\right)
$$

It should be noted that such a model of the impedance matrix corresponds in the time domain to a non-causal system (in particular the static impedance is then complex), and that the models that will be constructed in section 3 all refer to the linear model of the soil impedance matrix (equation $(8)$ without $[B]$ ). However, the comparisons with the soil impedance matrix computed using other methods (here, the Boundary Element - BE - method) are performed after correction with $[B]$.

\section{APPROXIMATE DETERMINISTIC MODELS OF THE SOIL IMPEDANCE MATRIX}

We briefly discuss in this section two approximate deterministic models of the soil impedance matrix: the lumped-parameter models and the hidden-variables models. They have in common that their parameters must be identified with respect to other results (Finite Element - FE - computation or Experimental measure, for example), and that they can form the basis of probabilistic models of the soil impedance matrix. However, some deficiencies of the former incline us towards the use of the latter, which will provide the basis of the nonparametric model of the soil impedance matrix. The last title of this section illustrates the construction of the hidden variables models with two examples of soils, the first one being used for the design case in section 5 .

\subsection{The lumped-parameter models}

The main type of simplified models [22] for the soil impedance matrix are Winkler springs models and Wolf's models, and can be grouped under the general denomination of lumpedparameter models. Some examples of these models can be found in [23, 24, 25, 26, 27, 28, 29]. They provide simple descriptions of the soil as equivalent models of springs, dashpots and masses, the values of which are identified from an impedance computed with an alternative method (e.g. FE). Also, when it is possible to define a normalized problem, they allow for a very fast computation of the soil impedance matrix. However they have several important discrepancies:

1. The structure of the model is specified a priori. The designer chooses the network of mechanical elements before the identification is performed. Therefore, the same input 
impedance matrix may lead to different models (compare, for example, the results in the references above, and see in particular [26]), and there is no certainty that the identified model is the best possible.

2. Each element of the impedance matrix is identified independently. This goes against physical sense, as the soil parameters influence all terms of the impedance matrix, or at least several. Besides, specific methods have to be introduced for the identification of the coupling terms of the impedance matrix (see for example [23]).

3. Identification of unphysical mechanical parameters. In most references above, the authors note that the identification of the mechanical elements yields negative values of the springs, dashpots and/or masses.

We now describe a more general deterministic model for the soil impedance matrix, that reproduces the advantage of simplicity of the lumped-parameter models, but without the above deficiencies.

\subsection{The hidden variables model}

In [30], a general framework was introduced for the construction of simplified models of boundary impedance matrices, enforcing their basic properties of causality and stability. However, the identification of these simplified models was described only element-by-element. More recently, this approach was fully generalized to the multidimensional case [31], with the simultaneous identification of the entire input impedance matrix. This model is briefly recalled below.

Using the notations of section 2.1, we suppose that we have computed or measured the impedance matrix $\left[Z_{s}(\omega)\right]$ of the foundation $\Gamma$ on a soil domain $\Omega_{s}$, with respect to a set of $n_{\Gamma}$ displacement fields $\left\{\boldsymbol{\Psi}_{\ell}\right\}_{1 \leq \ell \leq n_{\Gamma}}$. The construction of the hidden variables model is based on the hypothesis that, besides the $n_{\Gamma}$ DOFs defined on the boundary $\Gamma$, it is possible to define $n_{h}$ "hidden" DOFs defined within the interior of $\Omega_{s}$, and matrices of mass $\left[M_{s}\right]$, damping $\left[D_{s}\right]$ and stiffness $\left[K_{s}\right]$, corresponding to these $n=n_{\Gamma}+n_{h}$ DOFs. Although it is not possible to define precisely the physical shape corresponding to the hidden DOFs, they can be seen as internal modes of vibration. The matrices of the hidden variables model are block-defined as

$$
\left[M_{s}\right]=\left[\begin{array}{ll}
{\left[M_{\Gamma}\right]} & {\left[0_{\Gamma h}\right]} \\
{\left[0_{\Gamma h}^{T}\right]} & {\left[I_{h}\right]}
\end{array}\right],\left[D_{s}\right]=\left[\begin{array}{ll}
{\left[D_{\Gamma}\right]} & {\left[D_{c}\right]} \\
{\left[D_{c}^{T}\right]} & {\left[d_{h}\right]}
\end{array}\right], \text { and }\left[K_{s}\right]=\left[\begin{array}{ll}
{\left[K_{\Gamma}\right]} & {\left[K_{c}\right]} \\
{\left[K_{c}^{T}\right]} & {\left[k_{h}\right]}
\end{array}\right]
$$

where $\left[M_{\Gamma}\right],\left[D_{\Gamma}\right]$ and $\left[K_{\Gamma}\right]$ are full $n_{\Gamma} \times n_{\Gamma}$ symmetric, positive-definite matrices, $\left[D_{c}\right]$ and $\left[K_{c}\right]$ are full $n_{\Gamma} \times n_{h}$ matrices, $\left[d_{h}\right]$ and $\left[k_{h}\right]$ are diagonal $n_{h} \times n_{h}$ positive-definite matrices, $\left[I_{h}\right]$ is the $n_{h} \times n_{h}$ identity matrix and $\left[0_{\Gamma h}\right]$ is the $n_{\Gamma} \times n_{h}$ null matrix.

Contrarily to the lumped-parameter models of the impedance matrix (see first item in section 3.1 , the specific form of the matrices $\left[M_{s}\right],\left[D_{s}\right]$ and $\left[K_{s}\right]$ is not chosen a priori. The existence of several equivalent triplets of these matrices for one given soil impedance matrix is discussed in detail in [31], and any triplet of full $n \times n$ positive-definite matrices $\left[M_{s}\right],\left[D_{s}\right]$ and $\left[K_{s}\right]$ is shown to be equivalent to a triplet in the form of equation (10), with the only hypothesis that the damping for the hidden DOFs be of the proportional type. In such a hidden variables model, the approximate soil impedance matrix $[\tilde{Z}(\omega)]$, with respect 
Table I. Thickness $h$, unit mass $\rho$, compressional and shear wave velocities $c_{P}$ and $c_{S}$ Young's modulus $E$, shear modulus $G$, Poisson's ratio $\nu$, and hysteretic damping coefficient $\beta$ of the layers and substratum of the two models of soil.

\begin{tabular}{|c|c|c|c|c|c|c|c|c|}
\hline \hline & $\begin{array}{c}h \\
{[\mathrm{~m}]}\end{array}$ & $\begin{array}{c}\rho \\
{\left[\mathrm{kg} / \mathrm{m}^{3}\right]}\end{array}$ & $\begin{array}{c}c_{P} \\
{[\mathrm{~m} / \mathrm{s}]}\end{array}$ & $\begin{array}{c}c_{S} \\
{[\mathrm{~m} / \mathrm{s}]}\end{array}$ & $\begin{array}{c}E \\
{[\mathrm{GPa}]}\end{array}$ & $\begin{array}{c}G \\
{[\mathrm{GPa}]}\end{array}$ & $\begin{array}{c}\nu \\
{[-]}\end{array}$ & $\begin{array}{c}\beta \\
{[-]}\end{array}$ \\
\hline Stiff Soil & & & & & & & & \\
\hline Layer 1 & 10.5 & 2400 & 2809 & 1235 & 10.10 & 3.66 & 0.38 & $5 \%$ \\
\hline Layer 2 & 10.5 & 2500 & 3721 & 1741 & 20.60 & 7.57 & 0.36 & $5 \%$ \\
\hline Layer 3 & 19.5 & 2400 & 3715 & 1577 & 16.60 & 5.97 & 0.39 & $5 \%$ \\
\hline Substratum & $\infty$ & 2500 & 3799 & 1992 & 26.00 & 9.92 & 0.31 & $5 \%$ \\
\hline Soft Soil & & & & & & & & \\
\hline Layer 1 & 10.5 & 2400 & 338 & 149 & 0.15 & 0.05 & 0.38 & $5 \%$ \\
\hline Layer 2 & 10.5 & 2500 & 448 & 210 & 0.30 & 0.11 & 0.36 & $5 \%$ \\
\hline Layer 3 & 19.5 & 2400 & 448 & 190 & 0.24 & 0.09 & 0.39 & $5 \%$ \\
\hline Substratum & $\infty$ & 2500 & 457 & 240 & 0.38 & 0.14 & 0.31 & $5 \%$ \\
\hline \hline
\end{tabular}

to the $n_{\Gamma}$ DOFs of the interface, is given by

$$
\begin{aligned}
& {[\tilde{Z}(\omega)]=-\omega^{2}\left[M_{\Gamma}\right]+i \omega\left[D_{\Gamma}\right]+\left[K_{\Gamma}\right]-} \\
&\left(i \omega\left[D_{c}\right]+\left[K_{c}\right]\right)\left(-\omega^{2}\left[I_{\Gamma}\right]+i \omega\left[d_{\Gamma}\right]+\left[k_{\Gamma}\right]\right)^{-1}\left(i \omega\left[D_{c}\right]+\left[K_{c}\right]\right)^{T} .
\end{aligned}
$$

The identification of the full matrices $\left[M_{\Gamma}\right],\left[D_{\Gamma}\right],\left[K_{\Gamma}\right],\left[D_{c}\right]$, and $\left[K_{c}\right]$, and of the elements of the diagonal matrices $\left[d_{h}\right]$ and $\left[k_{h}\right]$ is described in [31], and will not be recalled here. However, it should be noted that it is performed on the entire impedance matrix, rather than on its elements, so that coupling is naturally taken into account. Also, the diagnosis of unphysical models is very natural, in comparison with lumped-parameter models, as the causality and stability of the soil impedance matrix are directly related to the positivity of $\left[M_{s}\right],\left[D_{s}\right]$ and $\left[K_{s}\right]$.

\subsection{The hidden variables model for two 4-layers soils}

We present in this section an example of the construction of the hidden variables models, for two 4-layers soils: a stiff one, and a softer one (see table I for the characteristics of the soil layers). Basically, they represent the same soil, but scaled so as to show two very different mechanical behaviors and illustrate fully the use of the hidden variables models. A BE software $[32,33]$ is used to compute the soil impedance matrix for that system with respect to a rigid circular foundation with a radius of $24.5 \mathrm{~m}$ (see figure 2). The stiff soil is the one that will be used in section 5 to illustrate the design of a building on an uncertain soil.

The identification of the impedance matrix for the first model is performed with 0 hidden variable (hence $\left[M_{s}\right]=\left[M_{\Gamma}\right],\left[D_{s}\right]=\left[D_{\Gamma}\right]$, and $\left[K_{s}\right]=\left[K_{\Gamma}\right]$ in that case), while 16 hidden variables are used for the softer model, corresponding to 4 hidden modes. The identification for both models was performed on the $[0,10] \mathrm{Hz}$ frequency range. The identified matrices are described in detail in appendix I and the modal properties of the second model, which are the $\left\{\omega_{\ell}\right\}_{1 \leq \ell \leq 4}$ and $\left\{\zeta_{\ell}\right\}_{1 \leq \ell \leq 4}$ such that $\left[d_{h}\right]=\operatorname{diag}\left(2 \omega_{\ell} \zeta_{\ell}\right)_{1 \leq \ell \leq 4}$, and $\left[k_{h}\right]=\operatorname{diag}\left(\omega_{\ell}^{2}\right)_{1 \leq \ell \leq 4}$, are summarized in table II. The soil impedance matrices computed using these two models are 

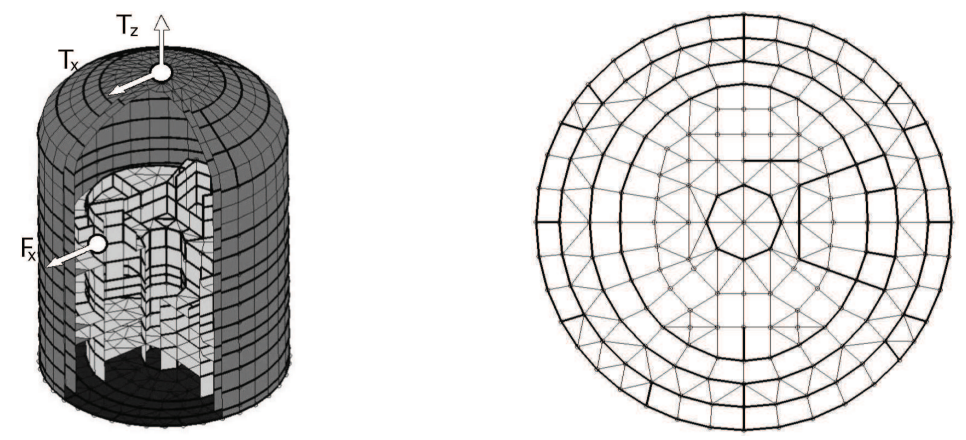

Figure 2. FE model of the building and BE model of the interface. The white arrows represent the sensors that are considered for the design: one horizontal $\left(T_{x}\right)$ and the vertical $\left(T_{z}\right)$ DOFs on top of the building and one horizontal $\left(\mathrm{F}_{\mathrm{x}}\right)$ DOF on a floor of the interior structure.

Table II. Modal properties of the hidden variables model for the soft soil.

\begin{tabular}{|c|c|c|c|c|}
\hline \hline Mode Number & 1 & 2 & 3 & 4 \\
\hline$\omega_{\ell}[\mathrm{rad} / \mathrm{s}]$ & 28.8 & 42.6 & 47.7 & 60.8 \\
\hline$\omega_{\ell}[\mathrm{Hz}]$ & 4.58 & 6.78 & 7.59 & 9.68 \\
\hline$\zeta_{\ell}[-]$ & 0.12 & 0.21 & 0.12 & 0.07 \\
\hline \hline
\end{tabular}

plotted and compared to the original soil impedance matrix, computed using the BE method, in figure 3. For brevity, only the shaking element, the rocking element, and the coupling between the shaking and the rocking are plotted.

\section{PROBABILISTIC MODELS OF THE SOIL IMPEDANCE MATRIX}

We concentrate in this section on the construction of probabilistic models of the soil impedance matrix. Two types of approaches exist: a parametric one and a nonparametric one. In the former, one constructs a probabilistic model for the parameters of the soil (mechanical parameters or boundary conditions for example) and derives the corresponding probabilistic model for the soil impedance matrix. In the latter, one considers directly the probabilistic model of the soil impedance matrix, that is constructed according to a given mean, a given level of variability, and a set of algebraic properties. We first review the main deficiencies of the parametric approach, and then we derive the nonparametric probabilistic models corresponding to the soils that were studied in the previous section.

\subsection{Parametric approach}

Several attempts have been made in the past at modeling the soil impedance matrix for a random soil, either based on a Stochastic Finite Element (SFE) formulation [34, 35, 36, $37,38,16,13,39,40,41]$, or on lumped-parameter models of the soil (Winkler spring models [42, 43, 44, 45, 46, 47] and Wolf's models [48, 49] in particular). The propagation of 

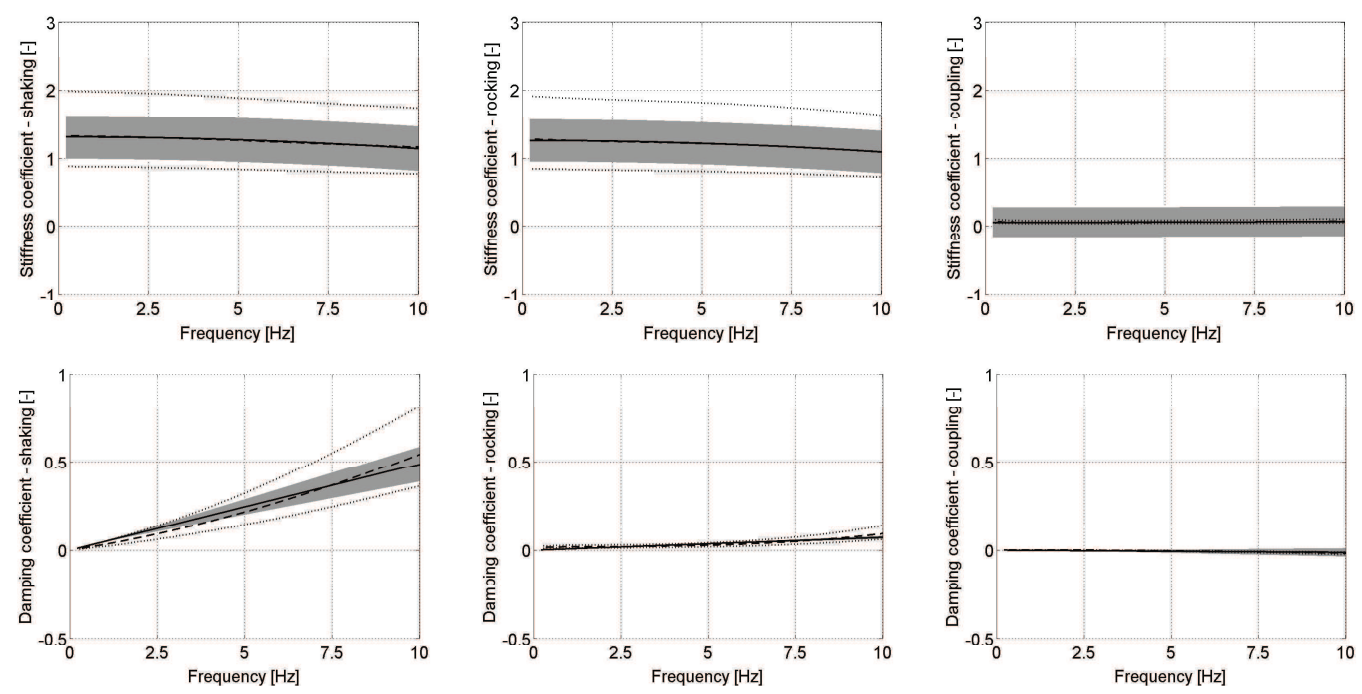

Figure 3. Dynamic stiffness (upper plots) and damping (lower plots) coefficients of the shaking (left plots), the rocking (center plots) and the coupling (rightmost plots) elements of the soil impedance matrix of the stiff soil, computed using a BE software (dashed line), and constructed using a hidden variables model with 0 hidden variable (solid line). The values computed for the extremal design (dotted lines) and the 95\%-confidence intervals computed using the nonparametric method (grey shades) are also plotted.

the uncertainty was performed either using the Monte Carlo method [18], which is very generic but usually time-consuming, or using approximate techniques [34, 35, 37, 42, 43, 46, 50, 51], that are often restricted to small levels of variability. These approaches include the classical Neumann expansion [52] and First-Order Second Moment [53] methods. Another technique consists in expanding the solution vector on a Polynomial Chaos basis [52], with, often, high induced computational costs. More examples of applications can be found in $[34,5]$. In these attempted models, the main difficulties concern:

1. The choice of the distribution for the random fields of soil parameters. When discussed at all, this choice is made rather arbitrarily (lognormal [13, 40, 41, 47], beta [40, 41, 46], gamma [54], truncated gaussian [36, 37, 38, 42, 43], for example), often only based on desired bounds for the values of the realizations of these parameters;

2. The choice of the correlation model for the random fields of soil parameters. This choice is made likewise groundlessly, almost always either as exponential [34, 36, 37, 38, 16, 39, 44, $46,50,54]$ or gaussian (squared exponential) [34, 13, 40, 41, 44]. However, both the choice of the correlation model and the estimation of the correlation lengths appear to be rather difficult experimentally (see [55] for an example of the difficulties encountered), and the influence of that choice on the overall response of the system is not fully understood. Some authors also advocate the use of fractal, or infinite-scale, models $[56,57]$. A more general discussion on the choice of the correlation model can be found in $[58,56]$;

3. The definition of appropriate boundary conditions. The use of FE techniques [59] for the modeling of an unbounded soil requires the definition of appropriate Absorbing Boundary 

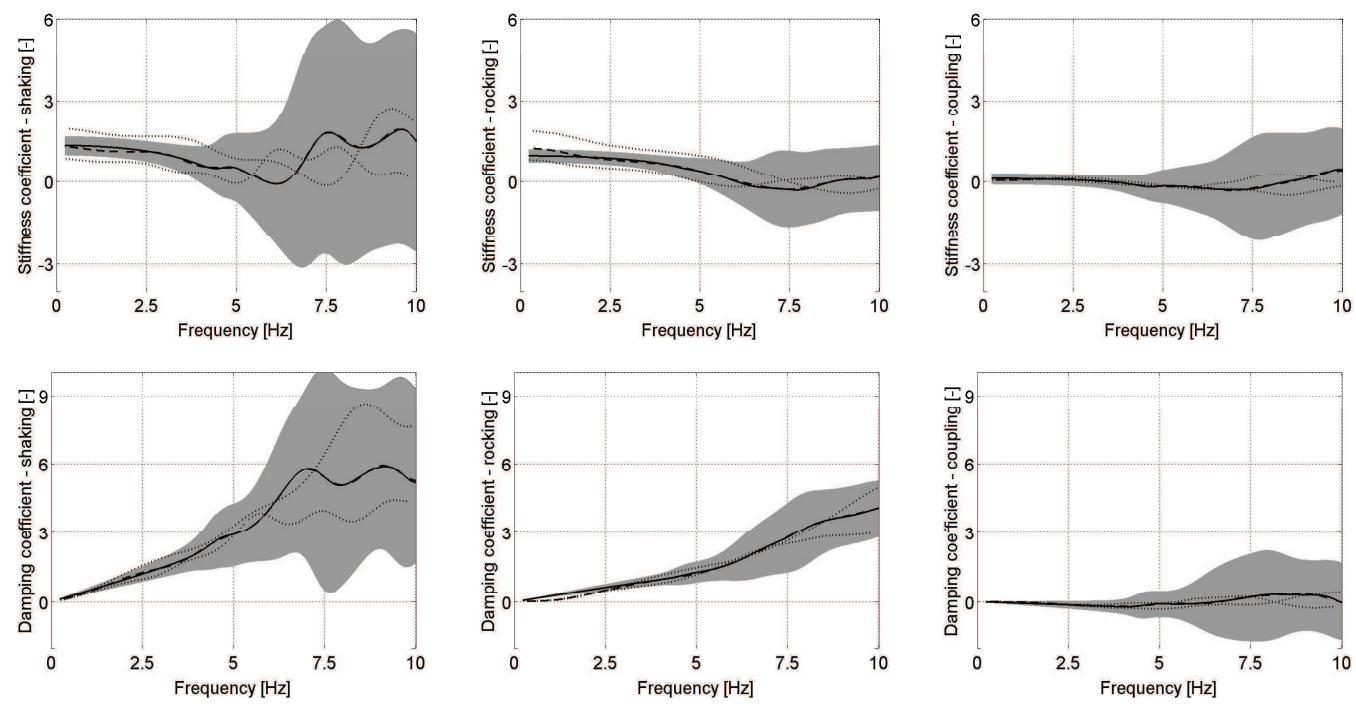

Figure 4. Dynamic stiffness (upper plots) and damping (lower plots) coefficients of the shaking (left plots), the rocking (center plots) and the coupling (rightmost plots) elements of the soil impedance matrix of the soft soil, computed using a BE software (dashed line), and constructed using a hidden variables model with 4 hidden variables (solid line). The values computed for the extremal design (dotted lines) and the 95\%-confidence intervals computed using the nonparametric method (grey shades) are also plotted.

Conditions (ABCs). Many types of ABCs exist [60], but their behavior in the case of a random soil is never assessed.

In the case of earthquake engineering, these difficulties are very hindering. We now discuss a novel approach for the modeling of the soil impedance matrix $\left[Z_{s}(\omega)\right]$ that takes into account both the natural heterogeneity of the soil parameters and the parametric uncertainties on these parameters, while avoiding the problems listed above.

\subsection{Nonparametric approach}

The general principle of the nonparametric approach consists in constructing the probabilistic model of the soil impedance matrix directly, bypassing the need to previously construct the probabilistic model of the soil parameters. It was originally introduced in structural vibration problems for the modeling of matrices of mass, damping and stiffness [61, 62], and recently extended to impedance matrices [31], through the use of the hidden variables models (see section 3.2). In this approach, the probabilistic model is constructed by enforcing that each realization of the matrix verify a given set of algebraic conditions. These conditions, for the impedance matrix, include that it be causal and stable. The mean of the impedance matrix is also imposed at the value of the deterministic model, and a level of fluctuations is selected.

The choice of that level of fluctuation can be made by comparison with experiments [63, $64,65]$. It can also be performed by comparison with numerical results conducted with an appropriate choice of the parametric probabilistic model $[66,67]$. In both cases, that level 
of dispersion is chosen by minimizing an appropriate function of the difference between the experimental measures (real-life or numerical) and the nonparametric model. However, for simplicity, we choose here to set the dispersion parameter equal for the three matrices $\left[\mathbf{M}_{s}\right]$, $\left[\mathbf{D}_{s}\right]$, and $\left[\mathbf{K}_{s}\right]$, and such that the $95 \%$-confidence interval lies, for all diagonal elements of the impedance matrix, just within the bounds corresponding to the extremal design (see figure 3 ). This choice corresponds approximately to $\delta=0.1$.

The practical construction of the nonparametric probabilistic model of the impedance matrix is done in the following way:

1. Computation of a deterministic model of the impedance matrix $\left[Z_{s}(\omega)\right]$ using any method.

2. Identification of the hidden variables model $\left(\left[M_{s}\right],\left[D_{s}\right]\right.$, and $\left.\left[K_{s}\right]\right)$ corresponding to $\left[Z_{s}(\omega)\right]$.

3. Computation of the nonparametric probabilistic models of $\left[\mathbf{M}_{s}\right],\left[\mathbf{D}_{s}\right]$, and $\left[\mathbf{K}_{s}\right]$, corresponding to these matrices, and using the Monte Carlo method.

4. Estimation, using equation (11), of the probabilistic model $\left[\mathbf{Z}_{s}(\omega)\right]$ of the impedance matrix.

Once the probabilistic model of the soil impedance matrix has been estimated, by the computation of a given number of Monte Carlo trials, the mean and confidence intervals can be derived for each frequency. It should be noted that the numerical cost of this method is very low, so that it is possible to estimate, at a given frequency, moments of high order. Indeed, the matrices that are considered in the nonparametric approach are very small, their size being defined by the number of DOFs on the foundation and the number of hidden variables. Each Monte Carlo trial of the impedance can therefore be computed very rapidly. On the contrary, SFE methods are defined on a basis including all physical DOFs in the soil, which implies large computational costs.

In figures 3 and 4 , the $95 \%$-confidence intervals are drawn for the stiff soil and the soft soil, respectively. Qualitatively, they seem to agree with the general experience that uncertainty increases both with the frequency and in the vicinity of resonances. These results were also backed up by similar models constructed using a SFE approach $[66,67]$. The impedance functions derived using the extremal design are also plotted on figures 3 and 4 . Although they seem qualitatively appropriate when there are little dynamic effects, such as in the case of the stiff soil, it is not so in the case of the soft soil. Indeed, for example for the rocking element of the impedance matrix, they predict more uncertainty in the low-frequency range than in the high-frequency range, which goes against physical sense.

The next section concentrates on the study of the seismic design of a building. It should be reminded that, as described in Sec. 2.2, no probabilistic modeling will be performed for the building nor the seismic load. If we were to do that, the main difficulty would lie in the assessment of the correlation between the probabilistic model of the soil impedance matrix, and that of the seismic load. By using equation (5) to solve the SSI system, we are considering the correlation in a perfect sense. However, this expression is only valid for the case of a vertically-propagating incident plane wave, and this hypothesis is probably far-fetched for a wave propagating through a heterogeneous soil. 

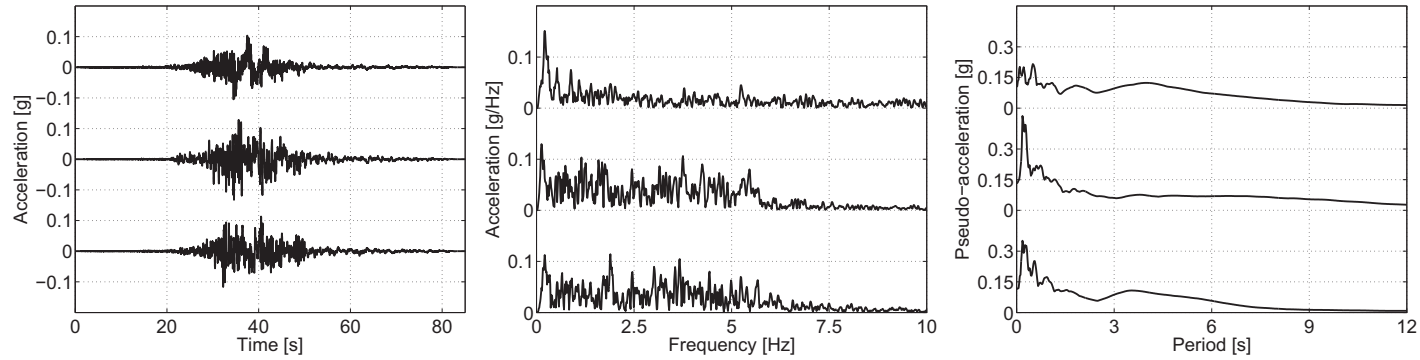

Figure 5. (a) Time history, (b) spectral content, and (c) pseudo-acceleration response spectrum of the East (bottom), North (center) and Vertical (up) components of the chosen seismic load.

\section{SEISMIC DESIGN OF A BUILDING RESTING ON AN UNCERTAIN SOIL}

\subsection{Model of the building}

The structure we consider here is a model of a reactor building (Fig. 2), with the shape of a cylinder closed by a semi-spherical top. The model is composed of a double wall, and the interior structure is also represented. The height of the building is $75 \mathrm{~m}$ and the radius of the cylinder is slightly below $25 \mathrm{~m}$. The FE model uses 2578 nodes and 7429 shell elements, and was computed using the open FE software OpenFEM [68]. The basis of the building lies 7.10 $\mathrm{m}$ below the soil level, and the hole dug in the soil is taken slightly larger than the radius of the foundation. The interface between the soil and the building is therefore a plane disc, and free field conditions are enforced on the sides of the cylinder over and below the soil level.

\subsection{Model of the seismic load}

We choose, for the seismic load, a moderate signal recorded on a site with stiff soil, on September $20^{\text {th }}, 1999$, during the Chi-Chi earthquake in Taiwan. The time history, spectral content, and pseudo-acceleration response spectrum for the three components of the input signal are presented in figure 5 .

\subsection{Seismic design of the building}

Once the probabilistic model of the soil impedance matrix $\left[\mathbf{Z}_{s}(\omega)\right]$ has been constructed (see section 4.2 and figure 3), equation (5) is solved for each Monte Carlo trial, and probabilistic models of the design quantities can be estimated. As discussed in section 2.3, we study here the maximum acceleration for three sensors: $\mathrm{T}_{\mathrm{x}}, \mathrm{T}_{\mathrm{z}}$, and $\mathrm{F}_{\mathrm{x}}$ (see figure 2), as well as the floor spectra, and the corresponding probabilistic quantities. In particular we consider and compare the values estimated using quantiles and confidence intervals, and those estimated using the extremal design method. They are plotted in figures 6 and 7. Convergence plots for the mean, the confidence intervals and the quantiles of the maximum acceleration at sensor $\mathrm{T}_{\mathrm{x}}$ are also presented in figure 8 .

The unreliable results obtained with the extremal method should first be noted. As expected mathematically, and foreseen in section 2.3, the nonlinear relation between the value of the impedance and that of the maximum acceleration in a given sensor means that a stiffer soil might unpredictably lead to a higher or lower maximum acceleration than a softer soil, 

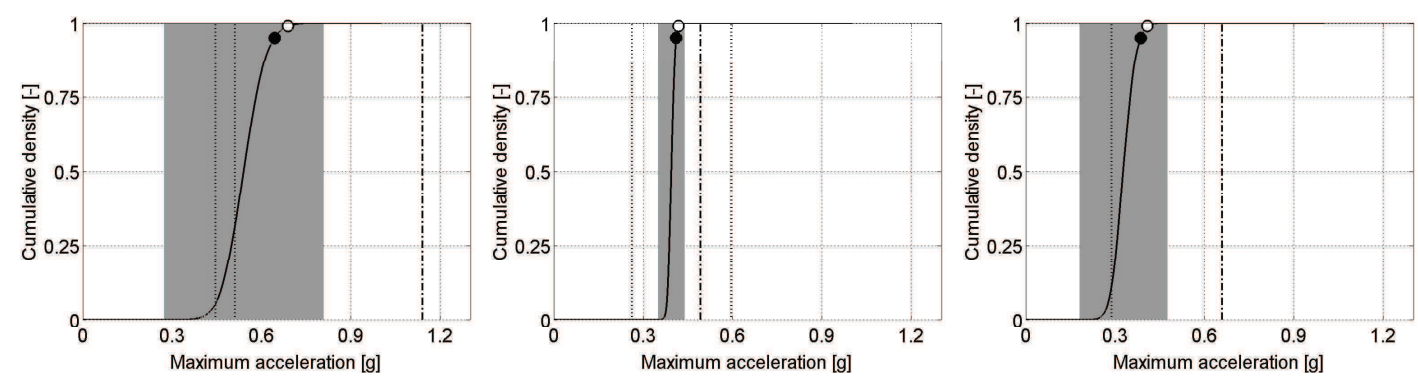

Figure 6. Estimation of the cumulative density function (solid line) of the maximum acceleration of sensors $\mathrm{T}_{\mathrm{x}}$ (left plot), $\mathrm{T}_{\mathrm{z}}$ (center plot), and $\mathrm{F}_{\mathrm{x}}$ (rightmost plot), and comparison of the $95 \%$-confidence interval (grey shade), the 95\%-quantile (black dot), and the values obtained with the extremal design (dotted lines). The 99\%-quantile (white dot) and the upper limit of the 99\%-confidence interval (dashdotted line) are also compared.
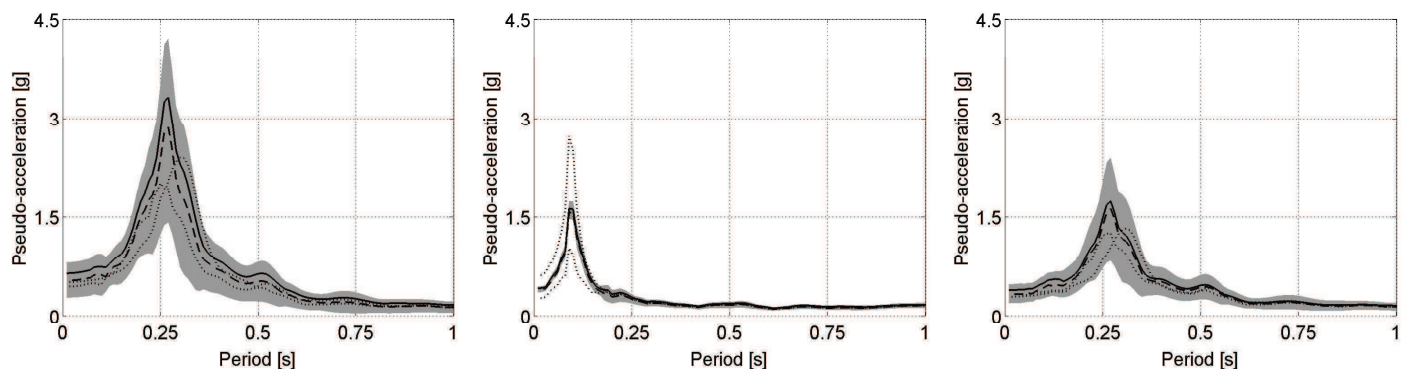

Figure 7. Floor spectra for sensors $\mathrm{T}_{\mathrm{x}}$ (left plot), $\mathrm{T}_{\mathrm{z}}$ (center plot), and $\mathrm{F}_{\mathrm{x}}$ (rightmost plot) for the design case (dashed line) and the extremal cases (dotted lines), and comparison with the 95\%-confidence interval (grey shade) and the $95 \%$-quantile (solid line) of the probabilistic design.
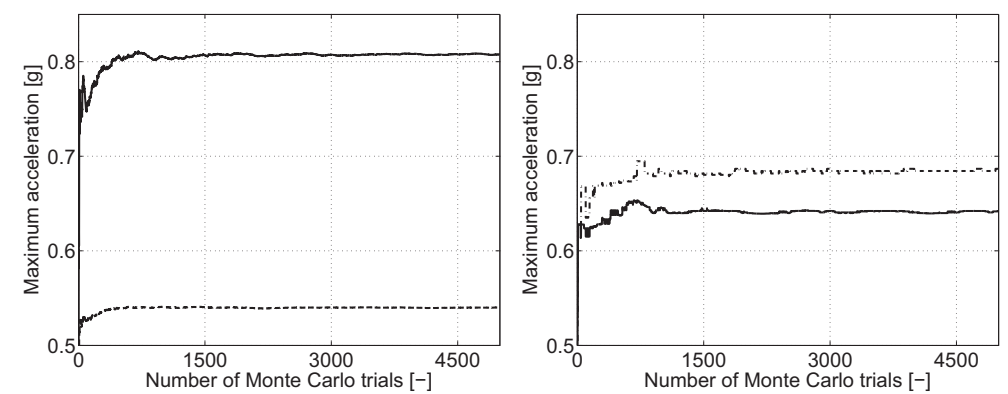

Figure 8. Convergence of the mean (left, solid dashed line), 95\%-confidence interval (left, solid line), and 95\%- and 99\%-quantiles (right, respectively in solid and dash-dotted line) of the maximum acceleration at sensor $\mathrm{T}_{\mathrm{x}}$. 
depending on the sensor and on the seismic load. In sensor $\mathrm{F}_{\mathrm{x}}$ for example (figure 6, right), the maximum acceleration estimated with the two soils of the extremal design method are the same (they are superimposed on the plot), but these maximum accelerations are not reached at the same instant. Likewise, and although they are not differentiated on the plots, for sensor $\mathrm{T}_{\mathrm{x}}$, the soft soil yields a higher maximum acceleration than the stiff one, while the contrary happens for sensor $\mathrm{T}_{\mathrm{z}}$. Therefore, this design method is not appropriate for the estimation of the uncertainty on the design of a structure on an uncertain soil, and should be abandoned.

Comparing then the 95\%-quantile and 95\%-confidence interval, it should be noted that the latter yields very conservative design values. This was again expectable, but is shown here to reach important values. In particular, for sensor $T_{x}$, the design maximum acceleration is lowered from around $0.8 \mathrm{~g}$ to around $0.65 \mathrm{~g}$ by considering the $95 \%$ - quantile instead of the 95\%-confidence interval. In the final design, this might represent substantial savings of material. The conservativeness of the design is even more blatant when considering the $99 \%$ quantile and the 99\%-confidence interval (see the case of the sensor $\mathrm{T}_{\mathrm{x}}$ in figure 6 for example). Equivalent remarks can be made about the floor spectra in figure 7 .

The consideration of the quantiles instead of the confidence intervals was made possible by the use of the nonparametric approach for the modeling of the soil impedance matrix. The equivalent with the parametric approach would, in most cases, be far too expensive. An exception to this might be the case of a low variability of the medium parameters, which usually cannot be considered for soils. Also, the nonparametric method does not require the identification of the probabilistic models of the soil parameters, which are usually difficult to estimate, and takes into account model errors, which are usually important in geomechanics. These two remarks make it a very advisable method for seismic design on uncertain soils.

\section{CONCLUSIONS}

We have presented in this paper the probabilistic construction of the soil impedance matrix for a SSI problem in earthquake engineering using a nonparametric method. Contrarily to the more classical parametric methods, this approach does not require the identification of the probabilistic model of the soil parameters, that are usually unavailable, and does not require the construction of ABCs specially designed for probabilistic unbounded media. Further, it allows, for a reasonable numerical cost, to estimate the quantiles of the quantities of interest, rather than the confidence intervals, that yield more conservative design values. Finally, possible modeling errors can also be taken into account with this method. The nonparametric approach is therefore very well suited for the seismic design of structures.

The main restriction to this conclusion arises from the assumed linearity of the soil, which is unrealistic for strong motion applications. However, it should be stressed that the hidden variables matrices which were described here can perfectly be used in a time-domain nonlinear analysis of the buildings to which they are coupled. This was not discussed here for lack of space but is a common feature with all lumped-parameter models of the impedance matrix the soil. Hence the only assumed linearity here is that of the soil.

This nonparametric method was applied to the design of a reactor building on a realistic soil. A concurrent design constructed with the classical extremal values approach was shown to be inadequate. The comparison of the results obtained with the nonparametric approach for quantiles and confidence intervals then indicated that the latter meant a high level of 
conservatism. The additional computational cost required for the computation of quantiles appears however reasonable when using the nonparametric method.

Following recent leads $[66,67]$, work is under way for the identification of the dispersion parameters for realistic soils. This last step will provide the means to perform a quantification of the impact of the soil uncertainty by comparison with the uncertainties associated with the seismic load and the building.

\section{APPENDIX}

I. Matrices of the hidden variables models

We describe here the hidden variables matrices that are used to construct the deterministic impedance matrices in figures 3 and 4 (solid lines). For symmetry reasons, and for both cases, only ten elements of the soil impedance matrix are non-zero (the diagonal elements and the coupling between the shaking in one direction and the rocking around the perpendicular direction), and the two horizontal directions are equivalent. Hence, for brevity, only the lines and columns relative to four DOFs will be presented. The DOFs correspond (in that order) to the translations along the first horizontal direction and the vertical direction, the rotation around the second horizontal direction, and the torsion around the vertical direction. The matrices $\left[M_{s}\right],\left[D_{s}\right]$ and $\left[K_{s}\right]$ are given by their sub-matrices, following the notations in eq. (10). It should be noted that, while some extra-diagonal element become negative, the diagonal elements remain positive, as well as the matrices themselves.

\section{I.1. Stiff soil}

In that case, there are no hidden variables, hence $\left[M_{s}\right]=\left[M_{\Gamma}\right],\left[D_{s}\right]=\left[D_{\Gamma}\right]$, and $\left[K_{s}\right]=\left[K_{\Gamma}\right]$.

$$
\begin{aligned}
{\left[M_{\Gamma}\right]=\left[\begin{array}{cccc}
0.45 & 0 & -0.04 & 0 \\
& 0.69 & 0 & 0 \\
& & 0.44 & 0 \\
& (\text { sym. }) & & 0.42
\end{array}\right] \times 10^{-4},\left[D_{\Gamma}\right] } & =\left[\begin{array}{cccc}
0.79 & 0 & -0.02 & 0 \\
& 1.12 & 0 & 0 \\
& & 0.12 & 0 \\
& (\text { sym. }) & & 0.09
\end{array}\right] \times 10^{-2}, \\
{\left[K_{\Gamma}\right] } & =\left[\begin{array}{cccc}
1.31 & 0 & 0.05 & 0 \\
& 1.75 & 0 & 0 \\
& & 1.25 & 0 \\
& (\text { sym. }) & & 1.07
\end{array}\right], \quad(12)
\end{aligned}
$$

\section{I.2. Soft soil}

In that case, there are 4 hidden modes, each with a multiplicity of 4 . The modal properties, which are the $\left\{\omega_{\ell}\right\}_{1 \leq \ell \leq 4}$ and $\left\{\zeta_{\ell}\right\}_{1 \leq \ell \leq 4}$ such that $\left[d_{h}\right]=\operatorname{diag}\left(2 \omega_{\ell} \zeta_{\ell}\right)_{1 \leq \ell \leq 4}$, and $\left[k_{h}\right]=$ $\operatorname{diag}\left(\omega_{\ell}^{2}\right)_{1 \leq \ell \leq 4}$, are summarized in table II (the $\omega_{\ell}$ should be taken in the formulas in rad/s). 
The sub-matrices are then:

$$
\begin{aligned}
& {\left[M_{\Gamma}\right]=\left[\begin{array}{cccc}
0.20 & 0 & 0.06 & 0 \\
& 0.16 & 0 & 0 \\
& & 0.15 & 0 \\
& \text { (sym.) } & & 0.40
\end{array}\right] \times 10^{-3},\left[D_{\Gamma}\right]=\left[\begin{array}{cccc}
0.09 & 0 & -0.01 & 0 \\
& 0.18 & 0 & 0 \\
& & 0.05 & 0 \\
& \text { (sym.) } & & 0.04
\end{array}\right]} \\
& {\left[K_{\Gamma}\right]=\left[\begin{array}{cccc}
2.82 & 0 & 0.27 & 0 \\
& 1.34 & 0 & 0 \\
& & 1.45 & 0 \\
& \text { (sym.) } & & 1.21
\end{array}\right]} \\
& \left.\left[D_{c}\right]=\left[\begin{array}{cccc}
-0.4 & 0 & 0 & 0 \\
0 & 0.3 & 0 & 0 \\
0 & 0 & 0 & 0.1 \\
0 & 0 & 0.1 & 0 \\
0.7 & 0 & -0.1 & 0 \\
0 & -0.5 & 0 & 0 \\
0 & 0 & 0.4 & 0 \\
0 & 0 & 0 & 0 \\
0.6 & 0 & 0.1 & 0 \\
0 & -0.8 & 0 & 0 \\
0.1 & 0 & -0.4 & 0 \\
0 & 0 & 0 & -0.2 \\
0 & 0.4 & 0 & 0 \\
0 & 0 & 0.1 & 0 \\
0 & 0 & 0 & 0.1 \\
0 & 0 & 0 & 0
\end{array}\right]^{T}\right]^{T} \quad\left[K_{c}\right]=\left[\begin{array}{cccc}
9.5 & 0 & 8.1 & 0 \\
0 & 4.6 & 0 & 0 \\
0 & 0 & 0 & -11.3 \\
-3.3 & 0 & 5.6 & 0 \\
-6.6 & 0 & 1.6 & 0 \\
0 & 16.5 & 0 & 0 \\
1.9 & 0 & 10.1 & 0 \\
0 & 0 & 0 & 16.2 \\
48.9 & 0 & 7.1 & 0 \\
0 & -9.7 & 0 & 0 \\
5.1 & 0 & -25.5 & 0 \\
0 & 0 & 0 & -18.3 \\
0 & -0.2 & 0 & 0 \\
8.9 & 0 & 1.8 & 0 \\
0 & 0 & 0 & 5.7 \\
-0.9 & 0 & 3.0 & 0
\end{array}\right]^{T}
\end{aligned}
$$

\section{REFERENCES}

1. Wolf JP. Dynamic soil-structure interaction. Prentice-Hall, Inc: Englewood Cliffs, NJ, 1985.

2. Clouteau D, Aubry D. Computational soil-structure interaction. In Boundary Element Methods for SoilStructure Interaction, Hall WS, Oliveto G (eds). Kluwer Academic, 2001.

3. Schuëller GI. A state-of-the-art report on computational stochastic mechanics. In Probabilistic Engineering Mechanics, 1997 ; 12(4):197-321.

4. Zerva A, Zervas V. Spatial variation of seismic ground motions: an overview. In Applied Mechanics Review, $2002 ; \mathbf{5 5}(3): 271-297$.

5. Manolis GD. Stochastic soil dynamics. In Soil Dynamics and Earthquake Engineering, 2002; 22:3-15.

6. Semblat JF, Kham M, Parara E, Bard PY, Pitilakis K, Makra K, Raptakis D. Seismic wave amplification: basin geometry vs soil layering. In Soil Dynamics and Earthquake Engineering, 2005; 25:529-538.

7. Favre JL. Errors in geotechnics and their impact on safety. In Computers and Structures, 1998; 67(1):3745. 
8. Lin YK, Kozin F, Wen YK, Casciati F, Schuëller GI, Der Kiureghian A, Ditlevsen OD, Vanmarcke EH Methods of stochastic structural dynamics. In Structural Safety, $1986 ; 3$ :167-194.

9. Ibrahim RA. Structural dynamics with parameter uncertainties. In Applied Mechanics Review, 1987 ; 40(3):309-328.

10. Luco JE, Wong HL. Response of a rigid foundation to spatially random ground motion. In Earthquake Engineering and Structural Dynamics, 1986; 14:891-908.

11. Harichandran RS, Vanmarcke EH. Stochastic variation of earthquake ground motion in space and time. In Journal of Engineering Mechanics, 1986; 112(2):154-174.

12. Deodatis G. Non-stationary stochastic vector processes: seismic ground motion applications. In Probabilistic Engineering Mechanics, 1996; 11:149-168.

13. Ghiocel DM, Ghanem RG. Stochastic Finite-Element analysis of seismic soil-structure interaction. In Journal of Engineering Mechanics, 2002; 128(1):66-77.

14. Labbé P, Noé H. Stochastic approach for the seismic design of nuclear power plant equipment. In Nuclear Engineering and Design, 1991; 129(3):367-379.

15. Desceliers C, Soize C, Cambier S. Non-parametric-parametric model for random uncertainties in non-linear structural dynamics: Application to earthquake engineering. In Earthquake Engineering and Structural Dynamics, 2004; 33(3):315-327.

16. Savin E, Clouteau D. Coupling a bounded domain and un unbounded heterogeneous domain for elastic wave propagation in three-dimensional random media. In International Journal for Numerical Methods ins Engineering, 2002; 54(4):607-630.

17. Chopra AK. Dynamics of structures: theory and applications to earthquake engineering. Prentice-Hall (2nd ed.), 2000.

18. Robert CP, Casella G. Monte Carlo statistical methods. Springer-Verlag, 1999

19. Krée P, Soize C. Mathematics of Random Phenomena: Random Vibrations of Mechanical Structures. D. Reidel Publishing Company, 1986.

20. Gazetas G. Analysis of machine foundation vibrations: state of the art. In Soil Dynamics and Earthquake Engineering, 1983; 2(1):2-42.

21. Sieffert JG, Cevaer F. Handbook of impedance functions. Surface foundations. Ouest Éditions, 1992.

22. Dutta SC, Roy R. A critical review on idealization and modeling for interaction among soil-foundationstructure system. In Computers \& Structures, 2002; 80(20-21):1579-1594.

23. Wolf JP, Somaini DR. Approximate dynamic model of embedded foundation in time domain. In Earthquake Engineering and Structural Dynamics, 1986; 14(5):683-703.

24. de Barros FC, Luco JE. Discrete models for vertical vibrations of surface and embedded foundation. In Earthquake Engineering and Structural Dynamics, 1990; 19(2):289-303.

25. Jean WY, Lin TW, Penzien J. System parameters of soil foundations for time domain dynamic analysis. In Earthquake Engineering and Structural Dynamics, 1990; 19(4):541-553.

26. Wolf JP. Consistent lumped-parameter models for unbounded soil: physical representation. In Earthquake Engineering and Structural Dynamics, 1991; 20(1):11-32.

27. Wolf JP. Consistent lumped-parameter models for unbounded soil: frequency-independent stiffness, damping, and mass matrices. In Earthquake Engineering and Structural Dynamics, 1991; 20(1):33-41.

28. Wolf JP, Paronesso A. Lumped-parameter model for a rigid cylindrical foundation embedded in a soil layer on rigid rock. In Earthquake Engineering and Structural Dynamics, 1992; 21:1021-1038.

29. Wu WH, Lee WH. Systematic lumped-parameter models for foundations based on polynomial-fraction approximation. In Earthquake Engineering and Structural Dynamics, 2002; 31(7):1383-1412.

30. Chabas F, Soize C. Modeling mechanical subsystems by boundary impedance in the finite element method. In La Recherche Aérospatiale (english edition), 1987; 5:59-75.

31. Cottereau R, Clouteau D, Soize C. Construction of a probabilistic model for impedance matrices. In Computer Methods in Applied Mechanics and Engineering, 2007; 196(17-20):2252-2268.

32. MISS software website. http://www.mssmat.ecp.fr/miss [9 November 2006].

33. Dominguez J. Boundary Elements in dynamics. Computer Mechanics Publications, 1993.

34. Baecher GB, Ingra TS. Stochastic FEM in settlement predictions. In Journal of the Geotechnical Engineering Division, Proceedings of the ASME, 1981; 107(GT4):449-463.

35. Righetti G, Harrop-Williams K. Finite element analysis of random soil media. In Journal of Geotechnical Engineering ASCE, 1988; 114(1):59-75.

36. Ghanem RG, Brzakala W. Stochastic Finite-Element analysis of soil layers with random interface. In Journal of Engineering Mechanics, 1996; 122(4):361-369.

37. Yeh C, Rahman M. Stochastic finite element methods for the seismic response of soils. In International Journal for Numerical and Analytical Methods in Geomechanics, 1998; 22(10):819-850.

38. Rahman MS, Yeh CH. Variability of seismic response of soils using stochastic finite element method. In Soil Dynamics and Earthquake Engineering, 1999; 18:229-245.

39. Liao S, Li J. A stochastic approach to site-response component in seismic ground motion coherency model. 
In Soil Dynamics and Earthquake Engineering, 2002; 22(9-12):813-820.

40. Nour A, Slimani A, Laouami N. Foundation settlement statistics via finite element analysis. In Computers and Geotechnics, 2002; 29:641-672.

41. Nour A, Slimani A, Laouami N, Afra H. Finite element model for the probabilistic seismic response of heterogeneous soil profile. In Soil Dynamics and Earthquake Engineering, 2003; 23(5):331-348.

42. Melerski E. Thin shell foundation resting on stochastic soil. In Journal of Structural Engineering, 1988; 114(12):2692-2709.

43. Chakraborty S, Sekhar Dey S. Stochastic Finite Element simulation of random structure on uncertain foundation under random loading. In International Journal of Mechanical Sciences, 1996; 38(11):12091218 .

44. Toubalem F, Zeldin B, Thouverez F, Labbé P. Vertical excitation of stochastic soil-structure interaction systems. In Journal of Geotechnical and Geoenvironmental Engineering, 1999; 125(5):349-356.

45. Breysse D, Niandou H, Elachachi S, Houy L. A generic approach to soil-structure interaction considering the effects of soil heterogeneity. In Géotechnique, 2004; 54(2):143-150.

46. Schevenels M, Lombaert G, Degrande G, Clouteau D. The wave propagation in a beam on a random elastic foundation. In Probabilistic Engineering Mechanics, 2006; 22(2):150-158.

47. Nedjar D, Hamane M, Bensafi M, Elachachi SM, Breysse D. Seismic response analysis of pipes by a probabilistic approach. In Soil Dynamics and Earthquake Engineering, 2007; 27:111-115.

48. Jin S, Lutes LD, Sarkani S. Response variability for a structure with soil-structure interactions and uncertain soil properties. In Probabilistic Engineering Mechanics, 2000; 15:175-183.

49. Lutes LD, Sarkani S, Jin S. Response variability of an SSI system with uncertain structural and soil properties. In Engineering Structures, 2000; 22:605-620.

50. Toubalem F, Thouverez F, Labbé P. Contribution in the study of a transfer function in a medium with random characteristics. In Applications of Statistics and Probability, Lemaire M, Favre JL, Mébarki A (eds). Balkema: Rotterdam, 1995:1197-1201.

51. Auvinet G, Mellah R, Masrouri F, Rodriguez JF. La méthode des éléments finis stochastiques en géotechnique. In Revue Française de Géotechnique, 2000; 93:67-79. In french.

52. Ghanem RG, Spanos PD. Stochastic Finite Elements: A Spectral Approach. Springer-Verlag, 1991.

53. Cornell AC. First-Order uncertainty analysis of soils deformation and stability. In Statistics and Probability in Civil Engineering, Proceedings of the 1st International Conference on Applications of Statistics and Probabilities to Soil and Structural Engineering, Lumb P. (ed), 1971:129-144.

54. Schevenels M, Lombaert G, Degrande G, Degrauwe D, Schoors B. The Green's functions of a vertically inhomogeneous soil with a random dynamic shear modulus. In Probabilistic Engineering Mechanics, 2006; 22(1):100-111.

55. Thompson EM, Baise LG, Kayen RE. Spatial correlation of shear-wave velocity in the San Francisco Bay Area sediments. In Soil Dynamics and Earthquake Engineering, 2007; 27:144-152.

56. Fenton GA. Estimation of stochastic soil models. In ASCE Journal of Geotechnical and Geoenvironmental Engineering, 1999; 125(6):470-485.

57. Fenton GA. Random field modeling of CPT data. In ASCE Journal of Geotechnical and Geoenvironmental Engineering, 1999; 125(6):486-498.

58. Klimeš L. Correlation functions of random media. In Pure and Applied Geophysics, 2002; 159:1811-1831.

59. Zienkiewicz OC, Taylor RL, Zhu JZ. The Finite Element method. Butterworth-Heinemann, 2005 (6th ed.).

60. Magoules F, Harari I (eds). Absorbing boundary conditions. In Computer Methods in Applied Mechanics and Engineering, 2006; 195(29-32):3551-3902.

61. Soize C. A nonparametric model of random uncertainties for reduced matrix models in structural dynamics. In Probabilistic Engineering Mechanics, 2000; 15:277-294.

62. Soize C. Maximum entropy approach for modeling random uncertainties in transient elastodynamics. In Journal of the Acoustical Society of America, 2001; 109(5):1979-1996.

63. Soize C. Non-gaussian positive-definite matrix-valued random fields for elliptic stochastic partial differential operators. In Computer Methods in Applied Mechanics and Engineering, 2006; 195:26-64.

64. Arnst M. Inversion of probabilistic models of structures using measured transfer functions. Ph.D. thesis, École Centrale Paris, France, 2007.

65. Arnst M, Clouteau D, Bonnet M. Inversion of probabilistic structural models using measured transfer functions. In Computer Methods in Applied Mechanics and Engineering, 2007; In Press.

66. Cottereau R. Probabilistic models of impedance matrices. Application to dynamic soil-structure interaction. Ph.D. thesis, École Centrale Paris, France, 2007.

67. Cottereau R, Clouteau D, Soize C. Approches paramétriques et non-paramétriques pour la modélisation probabiliste des matrices d'impédance. In Actes du 8ème Colloque National en Calcul des Structures, Combescure A, Boisse P, Sabourin F. (eds), 2007. In french.

68. OpenFEM software website. http://www-rocq.inria.fr/OpenFEM/ [2 December 2006]. 